\title{
Clinical and dermoscopic features of nevi in patients with psoriasis
}

\author{
Natalia Glebovna Ilina ${ }^{1 *}$, Yulia Mihailovna Krinitsuna ${ }^{1,2}$, Aleksey Viktorovich Taganov ${ }^{3}$, \\ Andrey Aleksandrovich Tulupov ${ }^{1,4}$, Irina Stanislavovna Kolesnikova ${ }^{5}$, Dmitry \\ Vladimirovich Yudkin ${ }^{5}$, Irina Gennadievna Sergeeva ${ }^{1,4}$ \\ ${ }^{1}$ Medical Department, Novosibirsk State University, Novosibirsk, Russia \\ ${ }^{2}$ Novosibirsk Research Institute of Regional Pathology and Pathomorphology, Novosibirsk, Russia \\ ${ }^{3}$ Scientific Research Centre 'Clinic of Dermatology', Moscow, Russia \\ ${ }^{4}$ Laboratory of translational brain research, The Institute International Tomography Center of the Russian Academy of Sciences, Novosibirsk, Russia \\ ${ }^{5}$ Chromosome Pathology Group, Institute of Molecular and Cellular Biology SB Russian Academy of Science, Novosibirsk, Russia
}

\begin{abstract}
The aim of the present study is to display the clinical and dermoscopic features of melanocytic nevi of more than $5 \mathrm{~mm}$ in diameter in psoriasis patients. A total of 32 patients with psoriasis (21 male, 11 female; average age 37.4) formed the first study group. In 22 patients (68.8\%), melanocytic nevi of $5 \mathrm{~mm}$ in diameter and bigger (total of 68 nevi) were clinically found. For a randomized trial, 100 people (21 male, 79 female; average age 27.8 ) without psoriasis were invited to form the second study group. Only 37 of them had nevi $\geqslant 5 \mathrm{~mm}$ in diameter (total of 60 nevi). Complete questionnaire, full body photometric skin examination, dermoscopy examination on the dermatoscope HEINE MINI 10X with 70\% ethyl alcohol immersion, skin type identification according to the Fitzpatrick classification, and nevi assessment according to $\mathrm{ABCD}$ and $\mathrm{ABC}$ rules were obtained for all recruited people. Our study showed that patients with psoriasis are more susceptible to melanocytic nevi of $>5 \mathrm{~mm}$ in diameter (68.8\%), while the result for the second group was $37 \%$. As for nevi of dysplastic criteria, we found $32 \%$ in psoriasis patients $v s .42 \%$ in the monitoring group. Moreover, we should bear in mind the influencing factors of skin phototype II and artificial insolation (e.g., tanning, PUVA (Psoralen and ultraviolet A), and narrowband phototherapy $311 \mathrm{~nm}$ ) obtained by the patients from the first group.
\end{abstract}

Keywords: melanocytic nevi; psoriasis; dermoscopy; melanoma

Citation: Ilina NG, Krinitsuna YM, Taganov AV, Tulupov AA, Kolesnikova IS, et al. Clinical and dermoscopic fea-tures of nevi in patients with psoriasis. J Surg Dermatol 2021; 6(1): 50; http://dx.doi.org/10.18282/jsd.v6.i1.50.

*Correspondence to: Ilina Natalia Glebovna, Medical Department, Novosibirsk State University, Novosibirsk, Russia; dr.natalia.ilina@gmail.com

Received: $24^{\text {th }}$ August 2020; Accepted: $6^{\text {th }}$ November 2020; Published Online: $23^{\text {rd }}$ November 2020

\section{Introduction}

Melanocytic nevi (MN) are benign melanogenic tumors, where melanocytes tend to develop into melanoma. Melanomas can arise from melanocytic nevi but much more often on intact skin. Multiple dysplastic or atypical
MN are considered to be an important factor associated with increased risk of melanoma ${ }^{[1,2]}$. It is believed that atypical nevi are precursor lesions for cutaneous melanoma. During teenage years (age 14-18), up to $25 \%$ of nevi are clinically atypical ${ }^{[3]}$. Ultraviolet (UV) radiation, both natural and artificial, is considered to be the

Copyright (C) 2021 Ilina NG, et al. This is an Open Access article distributed under the terms of the Creative Commons Attribution-Non Commercial 4.0 International License (http://creativecommons.org/licenses/by-nc/4.0/), permitting all non-commercial use, distribution, and reproduction in any medium, provided the original work is properly cited. 
main risk factor of melanocytic dysplasia appearance and further melanoma development ${ }^{[4]}$. Patients with psoriasis are of greatest interest because they receive UV therapy such as narrowband phototherapy $311 \mathrm{~nm}$, PUVA (Psoralen plus UVA), and excimer laser therapy ${ }^{[5]}$. Besides that, they take retinoids (which increase their sensitivity to UV radiation) and methotrexate (which has an immunosuppressing and cytostatic effect), further increasing the risk of skin melanoma appearance. Melanoma and Epstein-Barr virus-associated lymphomas in psoriasis patients taking methotrexate have been reported ${ }^{[6]}$.

Recently, a systematic literature review with meta-analysis was performed. The conclusion was that there is a slightly increased risk of some cancers (e.g., upper aero-digestive tract-, liver-, lung-, pancreatic-, and urinary tract cancers), with the most increased risk for skin carcinoma but no increased risk for melanoma ${ }^{[7]}$.

A literature presented one case of a psoriasis patient suffering from metastatic melanoma which regressed spontaneously without any treatment. The patient had psoriasis associated with HLA-Cw6 (human leukocyte antigen). So, there is a possible relationship between psoriasis, HLA-Cw6, and spontaneous melanoma regression; in that, psoriasis as an immune-mediated disease can play a protective role against malignant melanoma ${ }^{[8]}$.

Nowadays, dermoscopy is considered to be the most efficient tool for early diagnosis of melanoma. It reduces the frequency of unreasonable biopsy ${ }^{[9,10]}$ and is efficient for nevi monitoring.

The purpose of the present study is to display clinical and dermoscopic features of melanocytic nevi (of diameter $5 \mathrm{~mm}$ and more) among patients with psoriasis.

\section{Materials and methods}

The first step of the study was to recruit 32 patients with psoriasis (21 male and 11 female patients with an average age of 37.4 years old). They formed the first study group. Out of the 32 patients, 22 of them (68.8\%) were clinically found with melanocytic nevi of $5 \mathrm{~mm}$ in diameter and bigger (total of 68 nevi).

For a randomized trial, 100 people (21 male and 79 female with an average age of 27.8 years old) without psoriasis diagnosis were invited. They underwent a detailed examination to form the second monitoring group. Only 37 of them had nevi $\geqslant 5 \mathrm{~mm}$ in diameter (total of 60 nevi). All patients from both groups were followed-up only with a one-time visit.

Complete questionnaire and full body photometry, examination on the dermatoscope HEINE MINI 10X with $70 \%$ ethyl alcohol immersion, skin type identification, and nevi assessment with $\mathrm{ABCD}$ and $\mathrm{ABC}$ rules were obtained for all patients of the two groups. $\mathrm{ABCD}$ assessment included the following criteria: asymmetry, border, color, and dermoscopic features.

\section{Ethics statement}

This study was approved by the ethics committee. Written informed consent for participation in the study was obtained from all participants. There were no conflicts of interests reported.

\section{Results}

The questionnaire revealed the number of risk factors for melanoma skin cancer. It was filled by the patients themselves during their one-time visit.

The $1^{\text {st }}$ and $2^{\text {nd }}$ skin phototypes associated with a greater risk of melanoma were identified in $1(4.5 \%)$ and $16(72.7 \%)$ patients with psoriasis, respectively, and 2 $(5.4 \%)$ and $22(59.5 \%)$ people in the second study group, respectively. The $3^{\text {rd }}$ skin type was identified in $5(22.7 \%)$ psoriasis patients and $13(35.1 \%)$ people in the second group. Thus, patients with psoriasis have the most cases of $2^{\text {nd }}$ skin type and are vulnerable to sun exposure.

Sunburn episodes, at the age up to 18 years old, increased the melanoma appearance factor in $11(50.0 \%)$ psoriasis patients and $20(59.5 \%)$ people from the second group. Only $2(9.1 \%)$ and $3(8.1 \%)$ people from each respective group reported of having ever lived in countries with higher insolation than Novosibirsk. Presently, due to job or hobby factor, a total of $6(27.3 \%)$ and 8 $(21.6 \%)$ recruited people in each group, respectively, spend so much time in the open air, and most of the patients in both groups (13 (59.1\%) and 25 (67.6\%), respectively) are highly exposed to sunlight for more than two weeks a year. Moreover, 5 (22.7\%) and 5 (13.5\%) people from each group, respectively, had attended sun tanning sessions.

Additional melanoma risk factors for psoriasis patients were due to methotrexate and phototherapy treatments, of which $27.3 \%$ of recruited psoriasis patients received narrowband phototherapy $311 \mathrm{~nm}$ treatment, 9.1\% received PUVA therapy, and 9.1\% received methotrexate. For some patients, several methods of treatment were applied and therefore these patients had one or more additional risk factors. Thus, $36.4 \%$ of patients with psoriasis have additional factors negatively associated with melanoma risk.

Special attention was paid on the use of SPF (sun protecting factor) products to reduce the risk of 
melanoma development ${ }^{[11]}$. Only $1(4.5 \%)$ patient suffering with psoriasis and $3(8.1 \%)$ recruited people from the second group used SPF products when exposed to the open air for more than $1 \mathrm{~h}$, whereas only $2(9.1 \%)$ patients from the first group and $10(27.1 \%)$ people from the second group used SPF products when intermittently exposed to sunlight (for example, sunbathing). It is worth mentioning that patients used sun protective products with low SPF coefficient of up to 30 . The low frequency of SPF products usage among psoriasis patients could be explained by insolation as a treatment unit.

When being assessed subjectively, 50\% of patients from the first group and $84 \%$ of people from the second group had a big nevi number. Besides that, $5(22.7 \%)$ and $18(48.6 \%)$ of them, respectively, reported on the nevi's change over the course of time, but none of them consulted a doctor for an examination. Only 1 person from both groups (4.5\% and $2.7 \%$, respectively) had family melanoma anamnesis.

\section{Clinical characteristics}

When being examined, the number, size, and location of nevi were assessed (Table 1). The figures show that the size and location of nevi in individuals from the first and second groups did not change greatly, but patients with psoriasis showed a greater number of multiple $\mathrm{MN}$ (up to 15 per person) (Figure 1).

\section{Dermoscopic characteristics}

Nevi asymmetry was assessed according to three parameters: color, structure, and border. In the first group, most of the examined nevi $(52$, or $76.5 \%)$ were symmetric by color while about 16 nevi $(23.5 \%)$ indicated an axial symmetry of the color. A total of 49 nevi $(72.1 \%)$ were symmetric by structure, 15 nevi $(22.1 \%)$ showed axial structure symmetry, and 4 nevi (5.8\%) were asymmetric. The border symmetry assessment of the nevi revealed the following: 47 symmetric nevi $(69.1 \%), 13$ nevi with an axial symmetry (19.1\%), and 8 asymmetric nevi (11.8\%).

As for the second group, symmetry along both axes was observed by: color, 43 (71.7\%); structure, 28 (46.7\%); and borders, 30 nevi (50.0\%).

In both groups, none of the examined nevi had rough pigmentation cutoff at the lesion border.

When assessing lesions, the following color variations were observed: white, red, light-brown, and dark-brown.
Lesions of blue-black or black color were not found. Polychromy was found in 5 cases $(7.4 \%)$, with 56 cases $(82.4 \%)$ having two colors and 7 cases $(10.2 \%)$ having only one color of nevi in patients from the first group, whereas $12(20.0 \%), 33(55.0 \%)$, and $15(25.0 \%)$ of the abovementioned nevi cases were found in people from the second group, respectively.

While assessing dermoscopic features, pigment network was found in all examined nevi. Typical pigment network-light-to-dark-brown colored, regularly meshed and narrowly spaced network distributed regularly, thinning out at the periphery-was found in 46 cases $(67.6 \%)$ of the first group and in 37 cases $(61.7 \%)$ of the second group of people. Atypical pigment network is characterized by a black, brown, or gray colored, irregularly meshed network distributed irregularly

Table 1. Clinical characteristics of melanocytic nevi $>5 \mathrm{~mm}$ in diameter among psoriasis patients and patients of the monitored group

\begin{tabular}{lcc}
\hline & $1^{\text {st }}$ group $N=22$ & $2^{\text {nd }}$ group $N=37$ \\
\hline Location & $28(41.2 \%)$ & $19(31.7 \%)$ \\
Back & $12(17.6 \%)$ & $15(25.0 \%)$ \\
Chest & $10(14.7 \%)$ & $3(5.0 \%)$ \\
Shoulder & $9(13.2 \%)$ & $5(8.3 \%)$ \\
Abdomen & $3(4.4 \%)$ & $8(13.3 \%)$ \\
Huckle & $3(4.4 \%)$ & $1(1.7 \%)$ \\
Crus & $2(2.9 \%)$ & $4(7.0 \%)$ \\
Face & $1(1.6 \%)$ & $1(1.7 \%)$ \\
Forearm & - & $2(3.0 \%)$ \\
Neck & - & $1(1.7 \%)$ \\
Buttock & - & $1(1.7 \%)$ \\
Foot & & \\
Number & $10(45.5 \%)$ & $25(67.5 \%)$ \\
1 nevus & $6(27.3 \%)$ & $7(18.9 \%)$ \\
2 nevi & $6(27.3 \%)$ & $5(13.6 \%)$ \\
$\geqslant 3$ nevi & & \\
Diameter & $53(77.9 \%)$ & $44(73.3 \%)$ \\
$5-7 \mathrm{~mm}$ & $9(13.2 \%)$ & $8(13.3 \%)$ \\
$8-9 \mathrm{~mm}$ & $6(8.9 \%)$ & $8(13.3 \%)$ \\
$\geqslant 10 \mathrm{~mm}$ & &
\end{tabular}




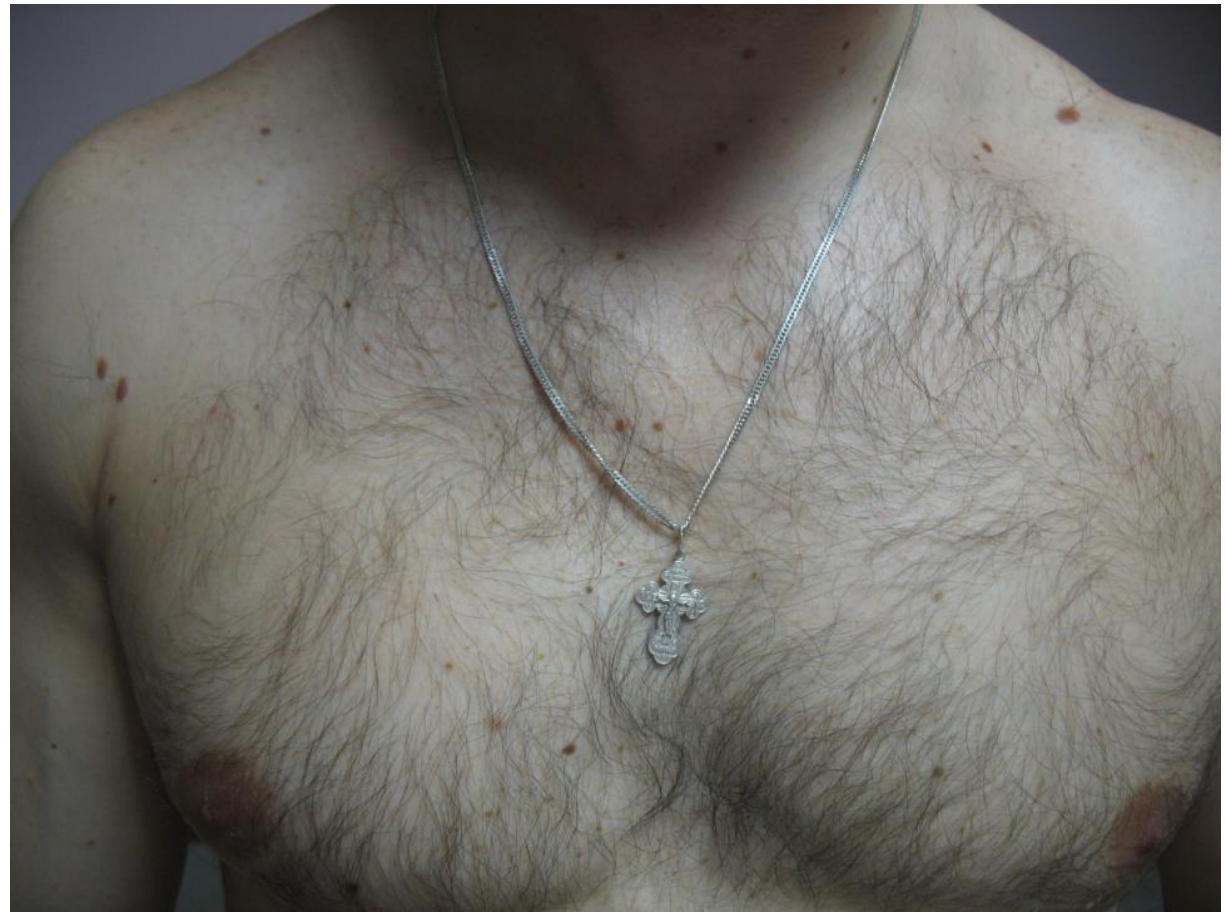

Figure 1. The chest area rated as a second location among psoriasis patients and patients of the monitored group

throughout the lesion. Lines of an atypical pigment network are often thickened and end abruptly at the periphery. Atypical network was found in 22 cases (32.4\%) from the first group and 22 cases $(36.7 \%)$ of the people from the second group, respectively.

A structureless area of light-brown or pink color was found in 29 cases $(42.6 \%)$ from the first group and in 30 cases $(50.0 \%)$ from the second group. Structureless areas were less pigmented compared to other areas, and often combined with pigment network areas or globules (Figure 2).

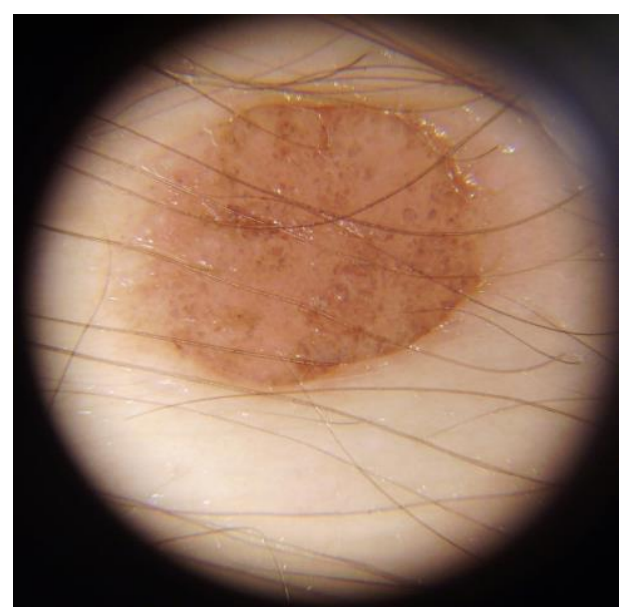

Figure 2. Dermal nevi with the combination of globules and structureless areas
Another frequent dermoscopic pattern is globules of dark-brown or light-brown color with round or oval structures, equal in size and distribution in the skin lesion. Globules were found in 47 nevi (69.1\%) of patients with psoriasis and 29 nevi (48.3\%) of the monitoring group. A net-globule combination was often observed (Figure 3).

It is known that acquired nevi have an age-related dynamic morphological pattern. First, an epidermal-dermal melanocytic nevus is formed due to the nevus cell proliferation along the border of epidermis and derma. In the course of time, nevi cells move further into the derma, thus forming a compound MN. When nevi cells immerse into the structure of the derma intensively, dermal $\mathrm{MN}$ is formed. A higher frequency of the globule structure nevi as a marker of the dermal MN among the patients of the first group can be explained by the average age of the recruited people, 37.4 years old, against the average age of 27.8 years old in the second group. Thus, a higher percentage of acquired nevi were observed in the phase of dermal MN.

Pseudopods are less frequently found in the examined skin lesions. Pseudopods are presented as linear structures of various thickness and curve endings, light- and dark-brown-colored, and heading in the radial direction from the center of the lesion to its periphery. Such areas were found locally in 4 nevi $(5.9 \%)$ of the first group and 9 nevi (15.0\%) of the second group (Figure 4). 


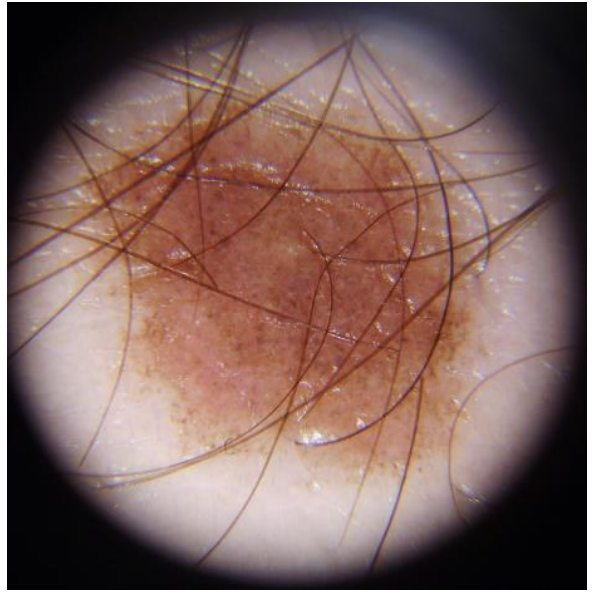

Figure 3. Large melanocytic nevi with globular pattern

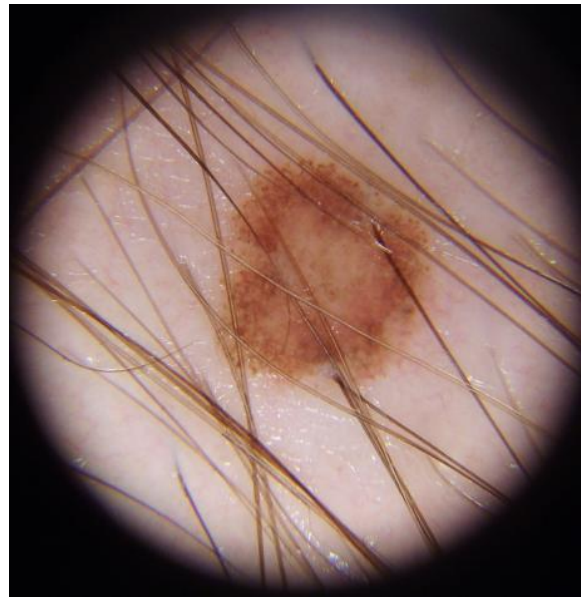

Figure 4. Large melanocytic nevi with pseudopods

Blotches - represented by areas of dark and dark-brown pigmentation, being quite major and structureless, and located in the center of the skin lesion-were found in 3 nevi $(4.4 \%)$ of the patients from the first group.

Forty-two nevi $(61.8 \%)$ in the first study group had two dermoscopic features, with 19 nevi (27.9\%) showing three dermoscopic features, and 6 nevi (8.8\%) revealing one feature. As for the second group, 29 (48.3\%), $19(31.7 \%)$, and $12(20.0 \%)$ cases were found, respectively. Only one nevus in the first group revealed four dermoscopic features. Combined structureless areas or a combination of pigment network and globules were often observed.

Using the ABCD rule, we calculated the Dermoscopy Score (DS) and stated that the index value of $>4.75$ corresponded to benign lesions, the value from 4.75 to 5.45 corresponded to potentially malignant lesions, and value of $>5.45$ to malignant lesions. We obtained index values of from 1 to 4.6 in 62 cases $(91.2 \%), 5.1$ in 4 nevi $(5.9 \%)$, and 5.6 in 2 nevi $(2.9 \%)$ from the first study group, and 54 (90.0\%), 4 (6.7\%), and $2(3.3 \%)$ nevi in the monitoring group, respectively.

$\mathrm{ABC}$ assessment included the same diagnostic criteria as in the ABCDE Rule (asymmetry, border, color, dermoscopic features, and skin lesion evolution), but another score system. The $\geqslant 4$ points assessment corresponds to malignant lesion. While assessing the nevi of patients with psoriasis, we obtained the following data: $\leqslant 3$ points for 66 cases $(97.1 \%)$, and thus their skin lesions were classified as benign; and 4 points for 2 nevi $(2.9 \%)$, hence these skin lesions were of malignant nature. In the second group, these values were 59 (98.3\%) for benign and $1(1.7 \%)$ for malignant lesions.

Only one nevus was diagnosed as a malignant lesion according to both $\mathrm{ABCD}$ and $\mathrm{ABC}$ rules in the group of psoriasis patients, and none in the monitoring group.

As for the Pehamberger analysis, melanocytic vi corresponded to the following criteria:

- The criterion of an epidermal-dermal nevus with an even pigment network was observed in $5(7.4 \%)$ and $9(15.0 \%)$ cases of the first and second group, respectively.

- Compound melanocytic nevus, which appears as reticular/granular model with a typical pigment network and hypopigmentation, were found in 36 $(52.9 \%)$ and $25(41.6 \%)$ cases, respectively.

- Dermal nevus with a typical dot/granular location, hypopigmentation, glomerular vessels were found in $5(7.4 \%)$ and $1(1.7 \%)$ cases, respectively.

- Dysplastic nevus which appears as reticular/granular model with a typical or atypical pigment network, regular or irregular dots/granules, regular streaks, blots and diffuse hypopigmentation were found in $22(32.4 \%)$ and $25(41.6 \%)$ cases, respectively (Figure 5).

\section{Discussion}

Dysplastic nevi are described as being in a continuum between common acquired nevi and melanoma because they are morphologically and biologically intermediate between these two entities. Since dysplastic nevi were initially being reported as histological lesions observed in melanoma-prone families, there were considerable debates about the definition of dysplastic nevi, the histological and clinical criteria used to define them, and their biological importance. In the dysplastic nevi's relationship to melanoma, it is not their primary role as 


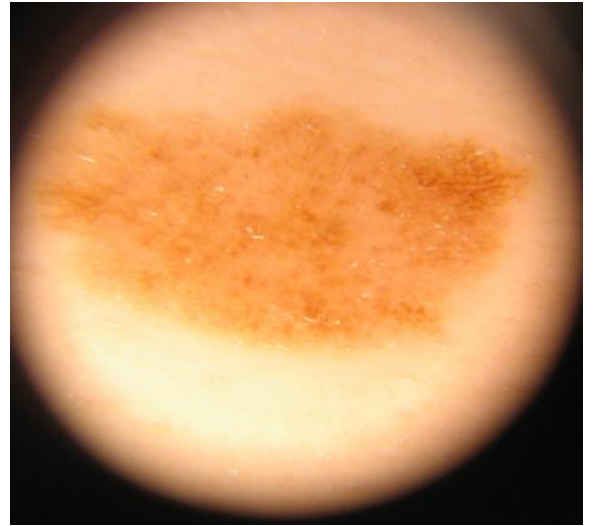

Figure 5. Dysplastic nevi characterized by Pehamberger criteria

precursor lesions for melanoma because of the rare transformation of any individual nevus to a melanoma ${ }^{[12]}$.

Overall, dysplastic nevi are relatively common with a frequency of about 10\% (range: 7\%-24\%) in Northern European populations ${ }^{[12]}$. Nevus with geographic shape and muddy borders, dominantly macular, red-to-brown-colored with $5 \mathrm{~mm}$ or more in diameter is a clinically dysplastic nevus. ABCD rules count for dysplastic nevi as well as for melanoma but the preferable diagnostic criteria for dysplastic nevi has been the "ugly duckling sign" (the only atypical lesion or the most atypical among other lesions). Pathohistological analysis can be the only diagnostic tool in the differential diagnosis between dysplastic nevus and melanoma in situ. So, surgical excision is the only therapy that should be done for dysplastic nevus with "ugly duckling sign"[13].

Some authors consider the term "dysplastic nevus" to be a misnomer and that it has to be abandoned. Preferably a specific diagnosis should be made based on dermoscopic pattern and, if this is not possible, on clinical or dermatoscopic signs alone, and the term "nevus, not otherwise specified" or "nevus with non-specific signs" should be used ${ }^{[14,15]}$. For further diagnosis, other clinical-diagnostic tools should be used and individual's melanoma risk should also be taken into account in decision of further treatment management.

According to all these up-to-date concepts of dysplastic nevi, we should consider $32 \%$ of "dysplastic naevi" by Pehamberger as not precursors for melanoma any more but only as nevi with structural changes, and use "ugly duckling sign" as the main dermoscopy rule to exclude melanoma.

Our study showed patients with psoriasis being more vulnerable to melanocytic nevi of $>5 \mathrm{~mm}$ in diameter $(68.8 \%)$, whereas the second group showed the result of $37 \%$. Dermoscopic picture of nevi in patients with psori- asis is symmetrical by color (76.5\%), border $(69.1 \%)$, and structure $(72.1 \%)$. In addition, $82.4 \%$ of nevi had two colors of light and dark-brown, and $69.1 \%$ had a globular structure of pigment.

Patients suffering from psoriasis had $32.4 \%$ cases of MN corresponding to Pehamberger dysplastic nevi, and only one case had the ABC and ABCD malignant symptoms.

In a very early study on 76 patients suffering from psoriasis, histological characteristics of melanocytic nevi within and near psoriatic lesions were investigated. Histopathologically examined nevi did not show any psoriatic alterations of the epidermis; the adjacent epidermis was free of psoriasis. Hence, that melanocytic nevi is a possible "protective" factor against psoriasis ${ }^{[16]}$, was discussed for the first time.

Further investigation showed that some of the cytokines such as interleukin (IL)-1 $\alpha$, IL-6, and tumor necrosis factor $(T N F)-\alpha$ gene expression have effects on melanogenesis, and they are also reported to be involved in psoriasis. In this study, patients with psoriasis showed a lower number of areas with nevi compared to the control group. This suggests the probable inhibiting effect of the proinflammatory cytokines in psoriasis skin on melanocyte growth, and/or progression to nevi ${ }^{[17]}$.

This statement was also supported by another study among patients with psoriasis under therapy with biologics. It showed that, the number of biologics used in treatment was a risk factor for a higher nevus count, whereas the psoriasis severity did not correlate with the number of nevi ${ }^{[18]}$. So, treatment with biologics influence the proinflammatory cytokine level and therefore can cause further melanocyte growth. This study also showed that the frequency of nevi in psoriatic patients was lower than in the control group (patients without psoriasis) as in our study.

Recent publication about development of new nevi in patients using immunosuppressive agents showed that therapy with biologics was associated with increased nevi counts and appearance of dermoscopy changes in nevi, but none of the changes and the subsequently excised nevi were malignant ${ }^{[19]}$.

Despite that, it was reported that a 51-year-old patient developed a de novo cutaneous malignant melanoma after a 30-month treatment with TNF-alpha-antagonists because of moderate-to-severe plaque psoriasis. But before biologics the patient became treatment with fumarates, cyclosporine A, methotrexate, and cream PUVA and UVB. Due to the fact that many patients received different treatment before biological treatment, it is hard to conclude that biological treatment was the only root cause for melanoma ${ }^{[20]}$. 
It is accepted, that PUVA is one of the risk factors for non-melanocytic skin cancer (squamous cell carcinoma), especially after 350 or more phototherapy sessions over a lifetime. Unlike risk of melanocytic skin cancer and genital skin cancer are not increased by PUVA alone. But additional factors as previous UV damage due to chronic sun exposure and sun burns, immunosuppressive treatment, increase cutaneous carcinogenesis through PUVA. In contrast, other artificial UV treatment as broadband UVB, narrowband UVB, and UVA1 has not yet been linked to cutaneous carcinogenesis probably due to lack of large follow-up studies ${ }^{[21]}$. One retrospective study including 92 patients affected by psoriasis under treatment with narrowband UVB or PUVA-therapy showed noteworthy number of non-melanoma skin cancer ${ }^{[2]}$.

Our research showed that $36 \%$ patients with psoriasis have additional risk factors for melanoma due to other treatments, with $27 \%$ received narrowband phototherapy $311 \mathrm{~nm}, 9 \%$ received methotrexate, and 9\% received PUVA-therapy. We have found no cases of malignancy in our study, but further follow up of the patients is necessary, as careful monitoring is crucial.

A systematic literature search performed on PubMed, Embase, and Cochrane databases using the keywords "Psoriasis AND Neoplasms" from 1980 to January 2012 showed possible increased risk of some solid cancers in psoriasis such as respiratory tract-, upper aerodigestive tract-, urinary tract-, and liver cancer, as well as increased risk of non-melanocytic skin cancer (squamous cell carcinoma and basal cell carcinoma), whereas the risk of melanoma is not increased. A higher risk of non-melanoma skin cancers, especially squamous cell carcinoma, that could be explained as cumulative effect of UV and photosensitive agents and other immunosuppressive therapy ${ }^{[23,24]}$.

Another study also showed no increased risk for melanoma, but for non-melanoma skin cancer. It was associated with PUVA, cyclosporine and anti-TNF treatment in psoriasis. It also suggests to take into consideration incidences of cancer in patients history before using biologic or imunnosuppressive therapy because of lack of studies in these patient groups ${ }^{[7]}$.

Only $9 \%$ of all patients with psoriasis who used sunscreen creams or underwent total skin examination with dermoscopy were classified as having prophylaxis of melanoma and other skin cancer. Low frequency of SPF products usage among psoriasis patients could be explained by insolation as a treatment unit.

\section{Conclusion}

Patients with psoriasis tend to have no increased risk of melanoma and probably tend to have less melanocytic nevi because of possible protective role of inflammatory cytokines on melanogenesis.

Under biological treatment or other immunosuppressive therapy any dermoscopic changes in melanocytic nevi should be carefully monitored as alternative to excision. Nevertheless, we should always pay attention to individual risks of both melanocytic and non-melanocytic skin cancers from previous treatment of UV or immunosuppressant such as methotrexate. Despite the fact that the influence of long term UV therapy on melanocytic nevi is yet to be studied, we should provide routine clinical examination and dynamic dermoscopy for melanocytic lesions in patients with psoriasis during UV and systemic treatment. We also should keep in mind the importance of prophylaxis and the use of the sunscreen products among patients of this group.

\section{Acknowledgements}

The study was financially supported by the Russian Science Foundation (project \# 14-35-00020) in the decision to submit the manuscript for publication.

\section{Conflict of interest}

The authors declare no potential conflict of interest with respect to the research, authorship, and/or publication of this article.

\section{References}

1. Rezze GG, Leon A, Duprat J. Nevo displásico (nevo atípico) (Portugese) [Dysplastic nevus (atypical nevus)]. An Bras Dermatol 2010; 85(6): 863-871. doi: 10.1590/ S0365-05962010000600013.

2. Cyr PR. Atypical moles. Am Fam Physician 2008; 78(6): 735-740.

3. Csoma Z, Erdei Z, Bartusek D, Dosa-Racz E, Dobozy A, et al. The prevalence on melanocytic naevi among schoolchildren in South Hungary. J Eur Acad Dermatol Venereol 2008; 22(12): 1412-1422. doi: 10.1111/j.14683083.2008.02887.x.

4. Narayanan DL, Saladi RN, Fox JL. Review: Ultraviolet radiation and skin cancer. Int J Dermatol 2010; 49(9): 978-986. doi: 10.1111/j.1365-4632.2010.04474.x.

5. Wong T, Hsu L, Liao W. Phototherapy in psoriasis: A review of mechanisms of action. J Cutan Med Surg 2013; 17(1): 6-12. doi: 10.2310/7750.2012.11124. 
6. Gross RL, Schwartzman-Morris JS, Krathen M, Reed G, Chang $\mathrm{H}$, et al. A Comparison of Malignancy Incidence among Psoriatic and Rheumatoid Arthritis Patients in a Large US Cohort Arthritis Rheumatol. 2014 Jun; 66(6): 1472-1481. doi: 10.1002/art.38385

7. Beyaert R, Beaugerie L, Van Assche G, Brochez L, Renauld JC, et al. Cancer risk in immune-mediated inflammatory diseases (IMID). Mol Cancer 2013; 12(1): 98. doi: 10.1186/1476-4598-12-98.

8. Pérez Ramírez S, Parra V, Avilés Izquierdo JA, Vicario JL, Martín M, et al. Metastatic melanoma with spontaneous regression, psoriasis and HLA-Cw6: Case report and a hypothesis to explore. Tumori 2014; 100(4): 144e-147e. doi: 10.1700/1636.17932.

9. Marghoob AA, Scope A. The complexity of diagnosing melanoma. J Invest Dermatol 2009; 129(1): 11-13. doi: 10.1038/jid.2008.388.

10. Goodson AG, Grossman D. Strategies for early melanoma detection: Approaches to the patient with nevi. J Am Acad Dermatol 2009; 60(5): 719-735. doi: 10.1016/ j.jaad.2008.10.065.

11. Mancebo SE, Hu JY, Wang SQ. Sunscreens: A review of health benefits, regulations, and controversies. Dermatol Clin 2014; 32(3): 427-438. doi: 10.1016/j.det.2014.03.011.

12. Goldstein AM, Tucker MA. Dysplastic nevi and melanoma. Cancer Epidemiol Biomarkers Prev 2013; 22(4): 528-532. doi: 10.1158/1055-9965.EPI-12-1346.

13. Dediol I, Bulat V, Živković MV, Marković BM, Šitum M. Dysplastic nevus-Risk factor or disguise for melanoma. Coll Antropol 2011; 35 (Suppl 2): 311-313.

14. Kittler H, Tschandl P. Dysplastic nevus: Why this term should be abandoned in dermatoscopy. Dermatol Clin 2013; 31(4): 579-588. doi: 10.1016/j.det.2013.06.009.

15. Hurwitz RM, Tavel ME. The mythical concept and untoward consequences of a diagnosis of dysplastic nevus: An overdue tribute to A. Bernard Ackerman, MD. Dermatol Pract Concept 2015; 5(1): 31-34. doi: 10.5826/dpc.0501a05.

16. Wollina U, Schaarschmidt H, Henkel U, Roth H, Barta U, et al. (German) [A combination of psoriasis vulgaris and nevus cell nevus-Clinical, histopathologic and histochemical findings]. Z Hautkr 1990; 65(4): 381-322, 385387.
17. Balato N, Di Costanza L, Balato A, Patruno C, Scalvenzi $\mathrm{M}$, et al. Psoriasis and melanocytic naevi: Does the first confer a protective role against melanocyte progression to naevi? Br J Dermatol 2011; 164(6): 1262-1270. doi: 10.1111/j.1365-2133.2011.10271.x.

18. Di Cesare A, Riitano A, Suppa M, Fidanza R, Zangrilli A, et al. Frequency of melanocytic nevi in psoriatic patients is related to treatment and not to disease severity. J Am Acad Dermatol 2013; 69(6): 947-953. doi: 10.1016/j.jaad. 2013.08.017.

19. Koseoglu G, Akay BN, Kucuksahin O, Erdem C. Dermoscopic changes in melanocytic nevi in patients receiving immunosuppressive and biologic treatments: Results of a prospective case-control study. J Am Acad Dermatol 2015; 73(4): 623-629. doi: 10.1016/j.jaad.2015.07.013.

20. Kowalzick L, Eickenscheidt L, Komar M, Schaarschmidt E. Prolongierte Psoriasistherapie mit TNF- $\alpha$-Antagonisten. Entwicklung des malignen Melanoms (German) [Long term treatment of psoriasis with TNF-alpha antagonists. Occurrence of malignant melanoma]. Hautarzt 2009; 60(8): 655-657. doi: 10.1007/s00105-008-1695-8.

21. Hofbauer G. Phototherapie und karzinogenese (German) [Phototherapy and carcinogenesis]. Hautarzt 2013; 64(5): 349-353. doi: 10.1007/s00105-013-2587-0.

22. Maiorino A, De Simone C, Perino F, Caldarola G, Peris K. Melanoma and non-melanoma skin cancer in psoriatic patients treated with high-dose phototherapy. J Dermatolog Treat 2016; 27(5): 443-447. doi: 10.3109/09546634.2015.1133882.

23. Pouplard C, Brenaut E, Horreau C, Barnetche T, Misery $\mathrm{L}$, et al. Risk of cancer in psoriasis: A systematic review and meta-analysis of epidemiological studies. J Eur Acad Dermatol Venereol 2013; 27(Suppl 3): 36-46. doi: 10.1111/jdv.12165.

24. Richard MA, Barnetche T, Horreau C, Brenaut E, Pouplard C, et al. Psoriasis, cardiovascular events, cancer risk and alcohol use: Evidence-based recommendations based on systematic review and expert opinion. J Eur Acad Dermatol Venereol 2013; 27(Suppl 3): 2-11. doi: $10.1111 / j d v .12162$. 